\title{
RESIDÊNCIAS MULTIPROFISSIONAIS EM SAÚDE NO ENFRENTAMENTO DA COVID-19: RELATO DE INTERVENÇÕES INTERPROFISSIONAIS
}

MULTIPROFESSIONAL RESIDENCES IN HEALTH TO FACE COVID-19: REPORT OF INTERPROFESSIONAL INTERVENTIONS RESIDENCIAS MULTIPROFESIONALES EN SALUD EN EL COMBATE DE LA COVID-19: RELATO DE INTERVENCIONES

INTERPROFESIONALES

Viviane Oliveira Mendes Cavalcante ${ }^{1}$

Diógenes Farias Gomes ${ }^{2}$

Thatianna Silveira Dourado ${ }^{3}$

Francisco Annielton Borges Sousa ${ }^{4}$

Marianne César Gomes ${ }^{5}$

Maria Alcineide Dias Araújo ${ }^{6}$

Palavras-chave:

Residência Multiprofissional em Saúde; Infecções por coronavírus; Pandemias.

Keywords:

Multiprofessional Residency in Health; Coronavirus Infections; Pandemics.

Palabras clave: Residencia Multiprofesional en Salud; Infecciones por Coronavirus; Pandemia.

Submetido: $16 / 11 / 20$

Aprovado: $08 / 12 / 20$

Autor(a) para Correspondência: Viviane Oliveira Mendes Cavalcante Rua João Brasilino do Nascimento, $n^{\circ} 461$ Campo dos Velhos Sobral - CE

CEP: 62.030-183

E-mail: viviolivermendes@gmail.com

\section{RESUMO}

Trata-se de um relato de intervenção que objetivou descrever as experiências de coordenadores, docentes e discentes de dois programas de residencias multiprofissionais, na implementação de intervenções para o enfrentamento da Covid-19, desenvolvidas sob a perspectiva da interprofissionalidade. A experiência ocorreu de março a novembro de 2020, a partir da Residência Multiprofissional em Saúde Mental e Residência Multiprofissional em Saúde da Família, da Escola de Saúde Pública Visconde de Saboia. Identificaram-se que as ações possibilitaram maior engajamento de residentes com profissionais de diversos serviços do Sistema Municipal de Saúde, com ênfase na Estratégia Saúde da Família e na Rede de Atenção Psicossocial, facilitando a colaboração interprofissional em saúde para a prestação de apoio psicossocial, vigilância em saúde e ações de promoção da saúde e prevenção de agravos. Desse modo, concluiu-se que a integração entre programas de residências e sistemas de saúde corresponde a uma relação que tece desafios e potencialidades no campo da interprofissionalidade e que semeia um potencial para a resolução da emergência de saúde pública por Covid-19.

\footnotetext{
1. Enfermeira. Mestra em Saúde da Família (UVA/Renasf). Coordenadora do Programa de Residência Multiprofissional em Saúde da Família da Escola de Saúde Pública Visconde de Saboia (ESP-VS). E-mail: viviolivermendes@hotmail. com ORCID: https://orcid.org/0000-0001-5229-8222

2. Enfermeiro. Mestre em Saúde da Família (UFC). Doutorando em Saúde Coletiva (UECE). E-mail: diogenesfgo@ gmail.com ORCID: https://orcid.org/0000-0002-2181-6012

3. Enfermeira. Mestra em Saúde da Família (UFC). Coordenadora do Programa de Residência Multiprofissional em Saúde Mental da Escola de Saúde Pública Visconde de Saboia (ESP-VS). E-mail: tathiannasilveira@hotmail.com ORCID: https://orcid.org/0000-0002-2135-5009

4. Psicólogo. Mestre em Saúde da Família (UFC). Docente da Escola de Saúde Pública Visconde de Saboia (ESP-VS). E-mail: annielton_b@yahoo.com.br 0RCID: https://orcid.org/0000-0003-0692-782X

5. Psicóloga. Residente Multiprofissional em Saúde Mental da Escola de Saúde Pública Visconde de Saboia (ESPVS). E-mail: mariannecesargomes@gmail.com 0RCID: https://orcid.org/0000-0001-6091-3790

6. Cirurgiã Dentista. Residente Multiprofissional em Saúde da Família da Escola de Saúde Pública Visconde de Saboia (ESP-VS).E-mail: alcineide.d.araujo@gmail.com ORCID: https://orcid.org/0000-0001-7706-4414
} 


\section{ABSTRACT}

This is an intervention report, which aimed to describe the experiences of coordinators, teachers, and students of two multiprofessional residency programs in the implementation of interventions to face Covid-19, developed from the perspective of interprofessionality. The experience took place from March to November 2020, from the Multiprofessional Residence in Mental Health and Multiprofessional Residence in Family Health, from the Escola de Saúde Pública Visconde de Saboia. We identified that these actions enabled greater engagement of residents with professionals from different services of the Municipal Health System, with emphasis on the Family Health Strategy and the Psychosocial Care Network, facilitating interprofessional collaboration in health to provide psychosocial support, health surveillance, and health promotion and disease prevention actions. Thus, we concluded that the integration of residency programs and health systems corresponds to a relationship that weaves challenges and potentialities in the field of interprofessionality, and that sows a potential for resolving the public health emergency by Covid-19.

\section{RESUMEN}

Se trata de un relato de intervención, que objetivó describir las experiencias de coordinadores, docentes y de los alumnos de dos programas de residencia multiprofesionales en la implementación de intervenciones para el combate de la Covid-19, desarrolladas bajo la perspectiva de la Interprofesionalidad. La experiencia ocurrió de marzo hasta noviembre de 2020, a partir de la Residencia Multiprofesional en Salud Mental y Residencia Multiprofesional en Salud familiar, de la Escuela de Salud Pública Visconde de Saboia. Se identificó que las acciones posibilitaron mayor participación de residentes con profesionales de diversos servicios del Sistema Municipal de Salud, con énfasis en la Estrategia de Salud familiar y en la Red de Atención Psicosocial, facilitando la colaboración interprofesional en salud para prestación de apoyo psicosocial, vigilancia en salud y acción de promoción de salud y prevención de agravios. De ese modo, se concluye que la integración entre programas de residencias y sistemas de salud corresponde a una relación que teje desafíos y potencialidades en el campo de la Interprofesionalidad y que siembra un potencial para resolución de la emergencia de salud pública por Covid-19.

\section{INTRODUÇÃ 0}

0 ano de 2020 está sendo marcado pela principal crise de saúde pública dos últimos cem anos. Até o mês de outubro, foram mais de 44 milhões de casos confirmados e 1.178 .475 mortes no mundo por Covid-19 (SARS-Cov2). Somente no Brasil, foram mais de 5,5 milhões de casos confirmados e 158.456 mortes ${ }^{1}$.

A pandemia revelou desafios organizacionais e estruturais dos sistemas de saúde de todos os países ao redor do mundo e pôs à prova os modelos privados, previdenciários e universais de saúde. Como esperado, os sistemas privatistas, como o dos Estados Unidos da América (EUA), diferentemente de países com sistemas universais (Brasil, Espanha, Itália e outros), apresentaram o menor desempenho nas ações preventivas e garantia do direito à saúde².

Desse modo, no Brasil, foram implementadas intervenções como a criação de hospitais de campanha, as testagens rápidas em serviços da Estratégia Saúde da Família (ESF) para a identificação de casos suspeitos e as campanhas para o incentivo ao isolamento social. Para compor essas intervenções, os profissionais foram acionados, sendo os residentes em saúde incluídos na estratégia “0 Brasil Conta Comigo - Residentes na área de Saúde por meio da Portaria GM $n^{0}$ 580, de 27 de março de 2020, a fim de promover, por meio de incentivo financeiro, a incorporação das ações de residentes de Programas multiprofissionais e uniprofissionais nos espaços estratégicos de enfrentamento da Covid-193.

Essa ação estratégica, então, suscitou, das instituições formadoras, a adesão dos Programas de Residências em Saúde aos planos locais de gestão do cuidado durante a pandemia, para o desenvolvimento de ações de prevenção e vigilância da Covid-19, como apoio psicológico, estímulo ao isolamento social, atenção hospitalar e monitoramento dos casos. Esses Programas foram importantes parceiros para qualificar e inovar os processos de assistência, formação e prevenção.

As residências multiprofissionais em saúde têm um papel fundamental na formação e na qualificação do profissional da saúde. Baseiam-se fundamentalmente no exercício da integralidade, a fim de propiciar as mudanças necessárias no modelo técnico-assistencial. A residência aprofunda os 
debates teórico-práticos e permite a construção de novos saberes entre as diferentes categorias de profissionais da saúde ${ }^{4}$.

Para tanto, este artigo busca descrever as experiências de coordenadores, docentes e discentes de dois programas de residências multiprofissionais na implementação de intervenções para o enfrentamento da Covid-19, desenvolvidas sob a perspectiva da interprofissionalidade.

\section{METODOLOGIA}

Trata-se de um relato de intervenção, descritivo, de caráter retrospectivo, ocorrido no período de março a novembro de 2020.

0 município de Sobral, Ceará, foi considerado como cenário da experiência, onde estão presentes as Residências Multiprofissionais em Saúde da Família (RMSF) e Residência Multiprofissional em Saúde Mental (RMSM), instituídas, respectivamente, em 2001 e 2013, pela Escola de Saúde Pública Visconde de Saboia (ESP-VS), instituição integrada à Secretaria de Saúde do município.

0s Programas de Residências contavam com 75 profissionais de diferentes categorias da saúde (educação física, enfermagem, farmácia, fisioterapia, fonoaudiologia, odontologia, psicologia, nutrição, serviço social e terapia ocupacional). Esses vivenciaram ações de enfrentamento da Covid-19 em serviços como hospital de campanha, Centros de Saúde da Família (CSF), farmácia de medicamentos especiais, Vigilância Epidemiológica, Vigilância Sanitária, Unidade de Acolhimento (UA), Centro de Atenção Psicossocial (CAPS) e sede administrativa da Secretaria Municipal da Saúde (SMS), além de estratégias virtuais como o plantão psicossocial e as lives realizadas pelo Instagram, facilitadas pelas ESP-VS.

Essas ações foram acompanhadas pelo corpo de docentes do Sistema Saúde Escola, de modo que estes realizaram a função de coordenadores e tutores, e por profissionais vinculados aos serviços da ESF e da Rede de Atenção Psicossocial (RAPS), que atuam como preceptores de categoria, assim como é previsto nos respectivos Projetos Pedagógicos de Cursos da Saúde da Família e Saúde Mental, a fim de proporcionar o exercício da prática e da colaboração interprofissional ${ }^{5-6}$.

Este relato foi consolidado a partir da sistematização das observações participantes de coordenadores, docentes e discentes, na implementação de ações

\section{"As residências} multiprofissionais em saúde se constituíram como um dos dispositivos para a operacionalização da educação interprofissional"

para o enfrentamento da Covid-19. Os resultados da experiência foram discutidos sob o referencial teórico da interprofissionalidade $\mathrm{e}^{7-8}$.

\section{RESULTADOS E DISCUSSÃO}

\section{Atividades desenvolvidas pelos profissionais da Residência Multiprofissional em Saúde Mental}

0 paradigma da atenção psicossocial se pauta na organização dos processos de trabalho a partir da colaboração interprofissional, pois compreende que é por intermédio da horizontalização dos saberes e da cooperação entre esses que é possivel construir práticas em saúde mental desinstitucionalizantes e produtoras de autonomia ${ }^{9}$. Desse modo, os serviços que compõem a RAPS devem possuir a colaboração interprofissional como uma das diretrizes para a organização do cuidado.

De acordo com Soares ${ }^{10}$, as residências multiprofissionais em saúde se constituíram como um dos dispositivos para a operacionalização da educação interprofissional. Contudo, essa modalidade de ensino na saúde tem enfrentado, ao longo do tempo, alguns desafios relacionados aos impactos de uma história de formação em saúde ordenada por especialidades, com a prática centrada em um trabalho individualizado e mecanizado.

0 contexto pandêmico decorrente da Covid-19 exigiu dos profissionais residentes um fortalecimento da integração e comunicação entre as diversas categorias profissionais para organizar um plano de cuidado para a minimização dos agravos. Com isso, observou-se o aumento da coesão dentro da equipe, com o desenvolvimento de novas práticas a partir do diálogo entre as profissões, técnicos e gestores, potencializando processos de trabalho a partir da colaboração interprofissional.

As adequações relacionadas aos processos de 
trabalho dos CAPS, devido à suspensão de grupos e reorientação dos atendimentos individuais, desencadearam a necessidade de mais encontros e diálogos entre os trabalhadores do serviço, técnicos, gestores e profissionais residentes. Desse modo, houve momentos de forte interação, desencadeando a reflexão crítica sobre os processos de trabalho e a construção de projetos de intervenções em resposta às fragilidades identificadas. Tal ação se revelou essencial e, como produto de reflexão, foi observada a importância desses diálogos permanentes no cotidiano do serviço, que se fortaleceram no curso da pandemia.

A partir da atuação direta com a atenção à crise em saúde mental durante a pandemia, percebeu-se 0 quanto o adoecimento não se restringe aos aspectos biológicos, mas está fortemente interligado ao contexto social. Também foram identificadas situações de crise relacionadas à instabilidade financeira, quando foram reduzidas as rendas de muitos usuários que as garantiam por meio de um trabalho autônomo. Isso repercutiu na garantia de seus direitos básicos, como alimentação e moradia.

Junto a essa fragilidade, as restrições relacionadas ao convívio social, em que espaços de escuta, de trocas afetivas e ressignificação proporcionados por atividades grupais, tanto na comunidade como nos grupos facilitados por profissionais do serviço, tiveram que ser suspensas, dificultando a cobertura de pessoas com sofrimento mental.

Esses desafios reforçam firmemente a integração entre os profissionais; no entanto, ainda observamos a resistência de alguns deles na tentativa de permanecer no modus faciendi do encaminhamento, sem diálogo com a equipe. Severo e Dimenstein ${ }^{11}$ analisam esse tipo de resistência a partir da possibilidade do resquício do predomínio do saber psiquiátrico sobre o cotidiano do serviço e o fato de as profissões de saúde ainda estarem pautadas em especialismos e com processos de trabalho fragmentados.

A Residência em Saúde Mental realizou uma aproximação com a população em situação de rua, mediante atividades semanais no Centro de Referência à População em Situação de Rua (Centro POP) realizadas por psicólogo, assistente social e profissional de educação física da residência junto aos profissionais do Centro de Atenção Psicossocial Álcool e outras Drogas (CAPS-AD). Nos momentos de educação em saúde, foram apresentados aos participantes os protocolos de biossegurança e o

\section{"Essas ações transcenderam um atendimento em equipe e proporcionaram uma maior organização em torno das necessidades de saúde locais"}

acesso aos Equipamentos de Proteção Individuais (EPI); além disso, foram orientados a ficar protegidos e atentos à saúde mental, entre outras temáticas. Esses momentos proporcionaram trocas de experiências entre os participantes e profissionais.

0 envolvimento e a articulação entre os diversos setores, com foco na construção de ações de educação em saúde para a população em situação de rua (PSR), foi fundamental para a condução das ações frente à pandemia. Foram considerados atores estratégicos para a organização das ações emergenciais da pandemia de Covid-19 para a PSR: Saúde, Assistência Social, Sociedade Civil, Segurança Pública e Órgãos de Garantias de Direitos.

Essas ações transcenderam um atendimento em equipe e proporcionaram uma maior organização em torno das necessidades locais de saúde. Todavia, os profissionais que participam de uma prática colaborativa necessitam de modelos de governança claros e objetivos e de protocolos de atendimento que favoreçam a interação para alcançar os objetivos propostos neles. Com isso, fazem-se necessários encontros regulares para promover as trocas de experiências e compartilhamento dos casos atendidos para qualificar a atenção.

Houve também a necessidade de alocação de alguns residentes para atividades vinculadas à gestão dos casos, de monitoramento e vigilância epidemiológica. Desse modo, profissionais da assistência social, psicologia e educação física foram orientados a fornecer apoio à Secretaria da Saúde e Coordenação da Atenção Primária.

Esse deslocamento dos residentes em saúde mental dos seus espaços originários de atuação desencadeou um distanciamento das atividades de contato direto com os usuários, ou seja, “os especialismos se desfizeram em prol do desenvolvimento de projetos comuns"11:343. No entanto, alerta-se que esses deslocamentos, sem um necessário acompanhamento pedagógico, podem 
gerar a sensação de despertencimento e provocar fragilidades no processo formativo.

0 afastamento dos residentes das suas áreas de concentração e o envolvimento com ações da gestão, mesmo que necessárias, proporcionaram, inicialmente, a não identificação com as atividades epidemiológicas. Isso foi trabalhado com um acompanhamento contínuo e contou com o apoio pedagógico de preceptores e docentes, que possibilitaram a revisão das práticas, buscando significados para a saúde mental e a prática colaborativa.

\section{Atividades desenvolvidas pelos profissionais da Residência Multiprofissional em Saúde da Família}

No contexto da pandemia, algumas estratégias foram pensadas para o enfrentamento da Covid-19, principalmente voltadas para o controle e a prevenção da disseminação do vírus. Dessas, algumas foram deflagradas pelos profissionais-estudantes vinculados aos programas de residência em saúde, a exemplo do monitoramento como tecnologia de coordenação do cuidado dos casos confirmados ou suspeitos da doença, implantado pelo Programa de Residência Multiprofissional em Saúde da Família de Rio Branco, Acre, de modo que a experiência promoveu a avaliação de risco e gravidade dos pacientes, identificou as necessidades de atendimento, seja presencial, teleconsulta ou atendimento de urgência e emergência, por meio de acompanhamento sistemático dos pacientes ${ }^{12}$.

No cenário da ESF de Sobral, os enfermeiros residentes em Saúde da Família inseridos nas unidades de saúde atuaram junto às equipes de Saúde da Família (eSF), inicialmente priorizando os atendimentos de pacientes sintomáticos respiratórios, o acolhimento dos casos de urgência que chegavam às unidades de saúde, o acompanhamento pré-natal das gestantes do território e visitas puerperais por meio de videochamada no aplicativo WhatsApp. A organização na farmácia e dispensação de medicamentos teve uma colaboração importante dos residentes de farmácia.

Outro apoio importante se refere a monitoramento diário dos pacientes sintomáticos com suspeita ou diagnóstico de Covid-19, semelhante à experiência de Rio Branco. Tal monitoramento foi realizado pelos cirurgiões-dentistas da equipe de saúde bucal dos CSF, com o apoio das demais categorias da Residência em Saúde da Família como, por exemplo, dentistas,

\section{"Monitoramento diário dos pacientes sintomáticos com suspeita ou diagnóstico de Covid-19"}

profissionais da educação física, psicólogos, fisioterapeutas e assistentes sociais, uma parte atuando presencialmente e outra parte apoiando em teletrabalho.

0 monitoramento era realizado por meio de ligações telefônicas diárias, por no mínimo 14 dias, ou por um período maior, quando observada a necessidade por persistência dos sintomas respiratórios. 0 monitoramento, por sua vez, permitiu a avaliação do quadro dos pacientes, bem como facilitou o diálogo com a equipe médica e de enfermagem, o que tornou a linha de cuidado mais estreita e resolutiva, pois a qualquer demanda havia o contato quase imediato e eram fornecidas orientações corretas e adequadas para o quadro desses pacientes.

No decorrer da evolução do quadro pandêmico e a diminuição do número de novos casos, emergiu o plano de retomada das atividades e com ele o retorno de parte e, posteriormente, da totalidade dos residentes aos seus respectivos territórios, cada um dentro de sua categoria; de modo que as equipes de residentes tiveram que adaptar-se à reorganização dos serviços e colaborar com o retorno periódico das atividades. 0s residentes atuaram junto às equipes de saúde na reconstrução dos fluxos de atendimento e na reorganização do acolhimento nas unidades, bem como realizaram o planejamento do retorno de grupos e a formulação das ações de promoção de saúde.

\section{Experiências na vigilância sanitária e vigilância epidemiológica}

Objetivando reduzir o risco da expansão da pandemia, os programas de residências apoiaram a vigilância sanitária por meio de abordagens em visitas domiciliares a pacientes com diagnóstico confirmado de Covid-19 que resistiam em cumprir as orientações da equipe da ESF para realizar 0 isolamento social ou quarentena e, assim, evitar a 
propagação do vírus.

A interação profissional com objetivos comuns permitiu a produção do cuidado ao enfrentamento da pandemia, de maneira compartilhada e oferecendo condições para a colaboração interprofissional entre esses, como: disposição de realizar as ações de enfrentamento nas visitas e diálogos com os pacientes, flexibilidade e proatividade diante das situações compartilhadas e postura para acolher 0 inesperado diante das solicitações da equipe da ESF. Essa postura favoreceu a corresponsabilização entre residentes e profissionais da vigilância sanitária e ESF.

Outras contribuições também ocorreram por meio de visitas a empresas e da abordagem aos ônibus que chegavam ao município, sempre com o intuito de orientar o cumprimento das medidas de isolamento social e quarentena.

A equipe que realizava as visitas era composta por dois assistentes sociais residentes, uma técnica de enfermagem e uma fisioterapeuta, sendo estas últimas oriundas da equipe da vigilância sanitária, sempre atuando quando solicitadas pela equipe da ESF, de forma colaborativa. No período, foram realizadas 138 abordagens domiciliares.

Outro cenário de atuação dos residentes foi a Vigilância Epidemiológica (VIGEP), que objetiva oferecer informações oportunas e fidedignas para as autoridades sanitárias tomarem as decisões no tempo certo. Diante do cenário da pandemia de Covid-19, a VIGEP contou com a contribuição de três residentes: dois fisioterapeutas e um nutricionista; também se juntaram à equipe da VIGEP duas enfermeiras e dois profissionais do Núcleo de Apoio Multiprofissional (NAM).

Fez-se necessária uma estreita articulação da VIGEP com a Atenção Primária à Saúde (APS) para a investigação e o rastreamento dos casos suspeitos e contatos, com posterior adoção de ações para reduzir o risco de disseminação da doença e fechamento dos casos que tiveram alta do tratamento, por meio de busca ativa junto à APS, de alimentação do sistema baseado em protocolos estabelecidos pelo município e da construção de mapas para o monitoramento dos casos suspeitos e diagnosticados.

\section{Experiências complementares}

No Brasil, o período da ditadura militar vetou o exercício do direito à cidadania. Somente com a redemocratização, na Constituição Federal de

\section{"O contexto atual provocado pela condição epidemiológica causada pela Covid-19 provocou e tencionou o ressurgimento dos sujeitos coletivos"}

1988, é garantida, nos princípios fundamentais da Constituinte, a Participação Social, regulamentada pela Lei n $8.142 / 90$, que dispõe sobre a participação da comunidade na Gestão do Sistema Único de Saúde (SUS). As decisões que afetam a vida da população brasileira passam a ser definidas a partir de discussões coletivas, promovendo o livre exercício da cidadania dos atores sociais, fortalecendo a democracia como essencial e legítima ${ }^{13}$.

0 processo de redemocratização do país e os movimentos sociais, como o Movimento de Reforma Sanitária, foram determinadores da maturação de um novo sujeito social brasileiro, consciente dos conceitos de sociedade e de cidadania, bem como da importância de sua prática cotidiana para a transformação da realidade ${ }^{14}$.

Nesse cenário, o SUS apresenta em sua agenda sanitária nacional uma nova forma de se fazer saúde, com dimensões políticas, tecnológicas e ideológicas. Suas ações e serviços constituem um sistema único, organizado, descentralizado, que presta atendimento integral sem prejuízos assistenciais e com participação popular ${ }^{15}$. A participação popular se consolida no SUS como estratégia de cogestão, estando, assim, no campo dos direitos políticos. A renovação das estruturas políticas e ideológicas levou a uma ruptura de paradigmas e fomentou o protagonismo dos próprios sujeitos, agora vistos como escritores e atores de uma história real.

0 contexto atual provocado pela condição epidemiológica causada pela Covid-19 provocou e tencionou o ressurgimento dos sujeitos coletivos. A pandemia fez com que o mundo sofresse paralisações das atividades econômicas e interrompesse as diversas formas de interação social.

0 isolamento social recomendado por autoridades sanitárias trouxe a necessidade de refletir sobre a condição de vulnerabilidade humana, em que todos passaram a ser dependentes e interdependentes de cuidados a o longo da vida. Cuidar envolve diversas tarefas, dentre elas: acolher, escutar, ser empático, 
zelar pelo bem individual e coletivo, mas como fazer isso quando fomos afastados de nossos pares, pelo menos da forma na qual estávamos acostumados, em que as relações pessoais envolviam encontros sociais presenciais e os afetos eram externalizados em forma de abraços, sorrisos e toques? Como manter a saúde mental e se sentir acolhido em meio ao cenário de angústias e incertezas? Diante de situações desafiadoras não temos todas as respostas, mas procuramos a cada instante enfrentar e desenvolver habilidades que nos levam a buscar estratégias de comunicação e interação que minimizem os danos sociais e afetivos causados pelo cenário epidemiológico.

A necessidade de distanciamento e isolamento social afetou, perceptivelmente, a organização de movimentos populares, principalmente nos territórios assistidos pelas residências aqui citadas. Desse modo, mecanismos de compartilhamento e empoderamento social sobre direitos sociais à saúde e métodos de prevenção à Covid-19 foram pautas de diálogos virtuais nos grupos de WhatsApp, tensionados por profissionais e líderes comunitários. Um dos desafios dessa estratégia foi a adesão e compromisso da comunidade devido às dificuldades de acesso à internet e ocupações cotidianas.

observou-se que o novo contexto das relações sociais, com restrições de contato e o direcionamento para o uso de meios virtuais, embora favorável ao incentivar a modernização e conectividade, trouxe consigo a necessidade de socialização entre as pessoas, característica fundamental do convívio humano. Isso, portanto, desencadeou uma série de casos de ansiedade identificados por busca telefônica ao CAPS.

Diante desse problema, uma estratégia complementar do CAPS, integrada aos profissionais da psicologia e serviço social das duas residências, foi o Plantão Psicossocial, que se fundamentou na assistência à pessoa com sofrimento mental, por meio de redes sociais (WhatsApp, Instagram e Facebook) e telefonemas. Tal estratégia se comprometeu numa relação entre a rede de saúde mental e de educação em saúde do município, proporcionando práticas interprofissionais em saúde, que caminharam para desfechos positivos, como a prevenção ao agravamento de síndromes de ansiedade e quadros graves de depressão.

Essas experiências proporcionaram 0 fortalecimento de ações integradas entre as residências, e delas com os profissionais das redes

\section{"0 isolamento social recomendado por autoridades sanitárias trouxe a necessidade de refletir sobre a condição de vulnerabilidade humana"}

de atenção, valorizando um trabalho integrado e comprometido com o bem-estar social. Para Reeves, Xyrichis e Zwarenstein ${ }^{16}$, o trabalho em saúde, diante das necessidades dos usuários, da família ou da comunidade, que, na maioria das vezes, são de difícil enfrentamento, exige a permanente articulação dos diferentes saberes e das práticas profissionais. É essa permanente interação que nos aproxima de um modelo de atenção à saúde mais integral, que é a meta que se busca alcançar nas práticas pedagógicas dos programas de residências.

Diante dos complexos desafios que surgiram, a partir das discussões foi possivel observar a importância do trabalho em equipe e a prática colaborativa como ferramenta de integração entre os diversos saberes que se constituem necessários para o aprimoramento do trabalho em saúde. Utilizando-se da prática colaborativa, tornouse factível a execução de ações propostas a partir de um planejamento participativo, em que os condicionantes sociais, culturais e biológicos foram considerados e amplamente discutidos.

0 envolvimento de diversos sujeitos e saberes oportunizou diálogos entre os campos disciplinares, tornando possivel aprender com o outro. Por fim, foi no cenário de incertezas que o exercício da interprofissionalidade ganhou força e reuniu esforços para transformar e promover a saúde das pessoas.

\section{CONCLUSÃO}

A colaboração das residências multiprofissionais em saúde, por meio de estratégias realizadas no enfrentamento da pandemia em curso, considerando a educação e a colaboração interprofissionais, possibilitou parcerias e qualificação do cuidado por meio da rede de atenção à saúde do município, fortalecendo o controle social e os protocolos clínicos e de manejo de casos suspeitos e confirmados de Covid-19, com vistas ao enfrentamento eficaz da pandemia. 
Entende-se a importância da colaboração interprofissional para o enfrentamento da pandemia, trabalhando diante de uma demanda eminente por atenção à saúde. Cabe destacar que a colaboração sistemática permitiu observar uma redução dos casos por meio do monitoramento, distanciamento social, quarentena e organização dos serviços.

A atual crise global sanitária pede inovação no modus faciendi e o fortalecimento do sistema de saúde para a realização de intervenções para - controle da pandemia de Covid-19. Destarte, deve-se incentivar a ampla e contínua colaboração das residências multiprofissionais com vistas ao trabalho colaborativo e interprofissional para o enfrentamento da pandemia do novo coronavírus, no intuito de, juntamente com o sistema de saúde, minimizar a expansão comunitária da doença.

0 desenvolvimento das ações relatadas neste artigo se deparou com desafios como a urgência na organização dessas estratégias, dificuldades de realização de ações que exigiram o uso de recursos tecnológicos e a distribuição igualitária de profissionais das residências diante do número elevado de ações que eram necessárias.

\section{CONTRIBUIÇÃO DOS AUTORES}

Viviane Oliveira Mendes Cavalcante contribuiu com redação, análise e revisão crítica do manuscrito. Diógenes Farias Gomes contribuiu com redação, análise e revisão crítica do manuscrito. Thatianna Silveira Dourado contribuiu com redação, análise e revisão crítica do manuscrito. Francisco Annielton Borges Sousa contribuiu com redação, análise e revisão crítica do manuscrito. Marianne César Gomes contribuiu com redação, análise e revisão crítica do manuscrito. Maria Alcineide Dias Araújo contribuiu com redação, análise e revisão crítica do manuscrito.

\section{REFERÊNCIAS}

1. World Health Organization. Covid-19. Brazil [home-page on the internet]. Genebra: WHO; 2020 [cited 2020 0ct 30]. Available from: https://covid19. who.int/region/amro/country/br

2. Conill EM. Sistemas comparados de saúde. In: Campos GWS, Minayo MCS, Akerman M, Drumond Júnior M, Carvalho YM, organizadores. Tratado de Saúde Coletiva. Rio de Janeiro: Hucitec, Fiocruz; 2016.
3. Brasil. Portaria $n^{0} 580$, de 27 de março de 2020. Dispõe sobre a Ação Estratégica “0 Brasil conta comigo - residentes na área da saúde" para o enfrentamento à pandemia do coronavírus (Covid-19) [document on the internet]. Brasilia (DF): Ministério da Saúde; 2020 [cited 2020 0ct 29]. Available from: https://www.in.gov.br/en/web/dou/-/portaria-n580-de-27-de-marco-de-2020-250191376

4. Salvador AS, Medeiros CS, Cavalcanti PB, Carvalho RN. Construindo a multiprofissionalidade: um olhar sobre a residência multiprofissional em saúde da família e comunidade. Rev Bras Cienc Saude [serial on the internet]. 2011 [cited 2020 0ct 30];15(3):32938. Available from: https://periodicos.ufpb.br/ojs/ index.php/rbcs/article/view/10834

5. Sobral. Projeto Pedagógico da Residência Multiprofissional em Saúde da Família. Escola de Saúde Pública Visconde de Saboia. Sobral: Prefeitura Municipal de Sobral; 2017.

6. Sobral. Projeto Pedagógico da Residência Multiprofissional em Saúde Mental. Escola de Saúde Pública Visconde de Saboia. Sobral: Prefeitura Municipal de Sobral; 2018.

7. Miller C, Freeman M, Ross N. Interprofessional practice in healht and social care: callenging the shared learning agenda. London: Arnold; 2001.

8. Reeves S. Why we need interprofessional education to improve the delivery of safe and effective care. Interface (Botucatu) [serial on the internet]. 2016 [cited 2020 Nov 10];20(56):185-96. Available from: https://scielosp.org/pdf/icse/2016. v20n56/185-197/en

9. Sousa FMS, Severo AKS, Félix Silva AV, Amorim AKMA. Educação interprofissional e educação permanente em saúde como estratégia para a construção de cuidado integral na Rede de Atenção Psicossocial. Physis [serial on the internet]. 2020 [cited 2020 Dec 10];30(1):e300111. Available from: http://www.scielo.br/scielo.php?script=sci arttext\&pid=S0103-73312020000100 $610 \& \operatorname{lng}=e n$

10. Soares GMM. Colaboração e Educação Interprofissional na Pós-graduação em Saúde: Estudo de caso da residência multiprofissional em saúde da família [dissertação]. Universidade Federal do Ceará; 2015

11. Severo AK, Dimenstein M. Processos de trabalho e gestão na estratégia de atenção psicossocial. Psicol Soc [serial on the internet]. 2011 [cited 2020 Nov 07];23(2):34-49. Available from: https://www.scielo.br/scielo.php?pid=S0102$\underline{71822011000200015 \& \mathrm{script}=\mathrm{sci} \text { abstract\&tlng }=\mathrm{pt}}$ 
12. Silveira RP, Leal 0, Soares PLS, Cruz LF, Modesto IDM, Batista LN, et al. Telemonitoramento da COVID-19 com participação de estudantes de medicina: experiência na coordenação do cuidado em Rio Branco, Acre. APS em Revista [serial on the internet]. 2020 [cited 2020 Nov 10]; 2(2):15161. Available from: https://apsemrevista.org/aps/ article/view/121

13. Brasil. Lei 8.142, de 28 de dezembro de 1990. Dispõe sobre a participação da comunidade na gestão do Sistema Único de Saúde (SUS) e sobre as transferências de recursos financeiros na área da saúde e dá outras providências [document on the internet]. Brasília (DF): Presidência da República; 1990 [cited 2020 Nov 09]. Available from: $\quad$ http://www.planalto.gov.br/ccivil 03/leis/l8142.htm

14. Coelho JS. Construindo a Participação Social no SUS: um constante repensar em busca de equidade e transformação. Saude soc [serial on the internet]. 2012 [cited 2020 Nov 05];21(Suppl 1):138-51. Available from: http://www.scielo.br/ scielo.php?script $=$ sci arttext \&pid $=S 0104-1290$ 2012000500012\&lng $=$ en

15. Gomes MCPA, Pinheiro R. Reception and attachment: integral practices in health care administration in large urban centers. Interface (Botucatu) [serial on the internet]. 2005 [cited 2020 Nov 08];9(17):287-301. Available from: https:// www.scielo.br/scielo.php?pid $=\mathrm{S} 1414-32832005000$ $\underline{200006 \& s c r i p t=s c i ~ a b s t r a c t}$

16. Reeves S, Xyrichis A, Zwarenstein M. Teamwork, collaboration, coordination, and networking: why we need to distinguish between different types of interprofessional practice. J Interprof Care [serial on the internet]. 2018 [cited 2020 Nov 09];2(1):13. Available from: https://www.tandfonline .com/doi/full/10.1080/13561820.2017.1400150
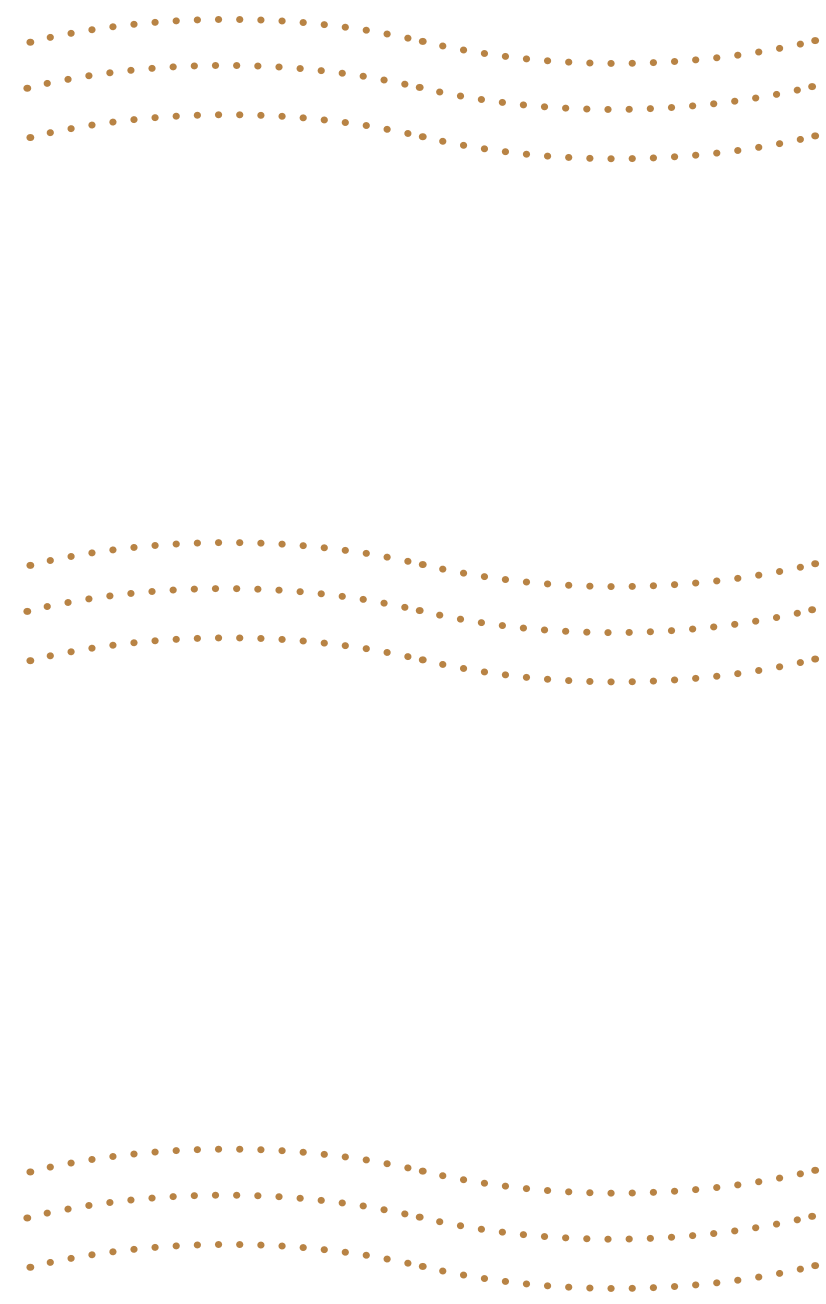